\title{
Lichen Scrofulosorum Following B.C.G.
}

\author{
R. G. EVANS and J. WARNER \\ From the Department of Paediatrics, and the Department of Dermatology, King's College Hospital, London S.E.5
}

Lichen scrofulosorum is a rare tuberculide seen in association with active tuberculous infection. With the increasing use of Bacillus Calmette-Guêrin (BCG) inoculation, several complications have been described but lichen scrofulosorum remains one of the rarest. A further case is therefore reported.

\section{Case Report}

A normal baby boy, birthweight $3855 \mathrm{~g}$. (8 lb. $8 \mathrm{oz}$.), was one of binovular twins. As their father had a past history of pulmonary tuberculosis, $0 \cdot 1 \mathrm{ml}$. BCG was given to both children intradermally in the deltoid region of the left arm on the third day of life. Four months later the child developed a rash around the BCG site which then spread to the limbs, trunk, and face.

Clinical examination at age 9 months showed a wellnourished healthy male infant with a rash chiefly on the trunk, but also scattered on the face and limbs. It consisted of brownish pinhead-sized papules. Very small depressed scars were seen at the site of some healed lesions, particularly around the area of BCG inoculation. The BCG site itself was well healed. There were no other abnormal clinical findings.

Investigations. Blood count and ESR normal. Serum protein electrophoresis showed a moderate increase in $a_{2}$ and $\gamma$-globulins. Chest $x$-ray film, normal. Mantoux test 1 in 1000 negative; 1 in 100 strongly positive $(0.5 \mathrm{~cm}$. raised centre, $1.5 \mathrm{~cm}$. erythema).

Biopsy of a lesion on the trunk showed a small collection of non-caseating tubercle follicles in the dermis. No acid-fast bacilli were seen.

Progress and treatment. When first seen, the rash was beginning to fade and scarring was minimal. In view of the good prognosis in lichen scrofulosorum when associated with tuberculosis, it was decided not to give any specific anti-tuberculous therapy.

However, over the next three months, the rash extended, particularly over the face and legs. Treatment with isoniazid $150 \mathrm{mg}$./day was therefore started at age 12 months. Two months later some improvement was noticed, and four months after starting treatment the rash was fading rapidly and no new lesions had appeared.

\section{Discussion}

Despite the increasing use of BCG, the frequency of generalized complications is as low as 1-2 per

\footnotetext{
Received October 14, 1966.
}

million (Horwitz and Meyer, 1957). It is therefore not surprising that lichen scrofulosorum is an extremely rare complication of BCG inoculation. In a review of dermatological complications of BCG (Dostrovsky and Sagher, 1963), of 33 cases, only 4 cases of widespread tuberculides, including lichen scrofulosorum, were found. Other cases of tuberculides have been described but none of them include lichen scrofulosorum.

The histology of lichen scrofulosorum is the same whether due to tuberculosis or BCG, and is characterized by the presence of non-caseating tubercles in the dermis.

In a true tuberculide, active tuberculous infection is always present. In this case, the BCG site appeared to be well healed, but it is known that an apparently well-healed scar may contain live tubercle bacilli in the midst of fibrous tissue, and that is probably what had occurred in our patient.

Why so few patients react in this way is not known. It has been postulated by Jabłońska, Rzucidło, and Fabiańska (1954) that skin reactions only occurred as the result of BCG activation of a latent tuberculous focus. This seems to be a possibility in one case described by Dostrovsky and Sagher (1963). However, in our case, BCG inoculation was done within three days of birth and there was no evidence of congenital tuberculous infection.

\section{Summary}

A case of a rare tuberculide, lichen scrofulosorum, is described following BCG inoculation.

Our thanks are due to Dr. R. H. Marten and Dr. C. E. Stroud for allowing us to publish this case.

\section{REFERENCES}

Dostrovsky, A., and Sagher, F. (1963). Dermatological complications of B.C.G. vaccination. Brit. F. Derm., 75, 181.

Horwitz, O., and Meyer, J. (1957). The safety record of B.C.G. vaccination and untoward reactions observed after vaccination. Fortschr. Tuberk.-Forsch., 8, 245.

Jabłońska, S., Rzucidło, L., and Fabiańska, L. (1954). Studies on the etiology of skin lesions of the tuberculous type developing under influence of BCG vaccination. I. Comparison of the properties of isolated strains with BCG strains and with BCGlike strains obtained from cases of tuberculosis luposa. (In Polish.) Przegl. Derm. Wener., 4, 145. 